\title{
On the origin of falling-tone chorus elements in Earth's inner magnetosphere
}

\author{
H. Breuillard ${ }^{1}$, O. Agapitov ${ }^{1,2}$, A. Artemyev ${ }^{1,3}$, V. Krasnoselskikh ${ }^{1}$, O. Le Contel ${ }^{4}$, C. M. Cully ${ }^{5}$, V. Angelopoulos ${ }^{6}$, \\ Y. Zaliznyak ${ }^{7}$, and G. Rolland ${ }^{8}$ \\ ${ }^{1}$ LPC2E/CNRS-University of Orléans, UMR7328, Orléans, France \\ ${ }^{2}$ National Taras Shevchenko University of Kyiv, Kyiv, Ukraine \\ ${ }^{3}$ Space Research Institute, RAS, Moscow, Russia \\ ${ }^{4}$ Laboratoire de Physique des Plasmas, CNRS/Ecole Polytechnique/Sorbonne Universités UPMC/Paris-Sud 11, \\ Saint Maur-des-Fossés, France \\ ${ }^{5}$ Department of Physics and Astronomy, University of Calgary, Calgary, Alberta, Canada \\ ${ }^{6}$ Institute of Geophysics and Planetary Physics, Department of Earth and Space Sciences, University of California, \\ Los Angeles, California, USA \\ ${ }^{7}$ Institute for Nuclear Research, Kyiv, Ukraine \\ ${ }^{8} \mathrm{CNES}$, Toulouse, France \\ Correspondence to: H. Breuillard (hbreuill@gmail.com)
}

Received: 17 March 2014 - Revised: 3 November 2014 - Accepted: 4 November 2014 - Published: 8 December 2014

\begin{abstract}
Generation of extremely/very low frequency (ELF/VLF) chorus waves in Earth's inner magnetosphere has received increased attention recently because of their significance for radiation belt dynamics. Though past theoretical and numerical models have demonstrated how risingtone chorus elements are produced, falling-tone chorus element generation has yet to be explained. Our new model proposes that weak-amplitude falling-tone chorus elements can be generated by magnetospheric reflection of rising-tone elements. Using ray tracing in a realistic plasma model of the inner magnetosphere, we demonstrate that rising-tone elements originating at the magnetic equator propagate to higher latitudes. Upon reflection there, they propagate to lower $L$ shells and turn into oblique falling tones of reduced power, frequency, and bandwidth relative to their progenitor rising tones. Our results are in good agreement with comprehensive statistical studies of such waves, notably using magnetic field measurements from THEMIS (Time History of Events and Macroscale Interactions during Substorms) spacecraft. Thus, we conclude that the proposed mechanism can be responsible for the generation of weak-amplitude falling-tone chorus emissions.
\end{abstract}

Keywords. Magnetospheric physics (plasma waves and instabilities)

\section{Introduction}

Since they were first revealed by ground-based observations (e.g., Storey, 1953; Allcock, 1957), chorus-type whistlermode waves, among the most intense natural emissions in the magnetosphere (e.g., Burtis and Helliwell, 1969; Tsurutani and Smith, 1977), have been studied intensively (see the reviews by Omura et al., 1991; Sazhin and Hayakawa, 1992). Spacecraft observations have shown that these waves exhibit discrete quasi-monochromatic elements from a few hundred hertz to a few kilohertz that propagate in two distinct frequency bands, scaling on the equatorial gyrofrequency $\Omega_{e, \text { equ }}$ (Burtis and Helliwell, 1969; Koons and Roeder, $1990)$. The lower band lies in the $\omega \approx 0.1-0.5 \Omega_{e \text {,equ }}$ range and the upper band in the $\omega \approx 0.5-0.8 \Omega_{e, \text { equ }}$ range with a wave power minimum around $0.5 \Omega_{e \text {,equ }}$ (Tsurutani and Smith, 1974). Chorus elements usually exhibit both rising $(\mathrm{d} \omega / \mathrm{d} t>0)$ and falling $(\mathrm{d} \omega / \mathrm{d} t<0)$ tones and sometimes short, impulsive bursts (see, e.g., Burton and Holzer, 1974; Anderson and Maeda, 1977; Hayakawa et al., 1984). Burtis and Helliwell (1976) demonstrated that rising tones are the most commonly observed chorus-wave elements (77\% of the observations), followed by falling tones (16\%). Constantfrequency tones, some combination of the above, named 
"hooks" (regular or inverted), and structureless narrowband elements were also found (Tsurutani and Smith, 1974).

Recent statistical studies of chorus waves using THEMIS (Time History of Events and Macroscale Interactions during Substorms) data (Li et al., 2011b, 2012) have confirmed the dominance of rising tones on both nightside and dayside and have shown that rising-tone wave amplitudes are typically much larger (a few tens to hundreds of picotesla) than those of falling tones ( $<50$ pT; see Li et al., 2011b, 2012). In these studies, rising and falling tones are found to occur preferentially in the region with lower values of $\omega_{\mathrm{pe}} / \Omega_{e \text {, equ }}$ (where $\omega_{\text {pe }}$ is the plasma frequency) from postmidnight to noon. Rising tones are mostly concentrated around large $L$-shells (>8); falling tones are mostly confined to small $L$-shells $(<8)$. These statistical analyses have also shown that rising tones are generally quasi-field-aligned $\left(\theta<30^{\circ}, \theta\right.$ is the wave-normal angle with respect to the local magnetic field direction), as found by Burton and Holzer (1974), Hayakawa et al. (1984), and Agapitov et al. (2011b), whereas falling tones are very oblique, as found by Cornilleau-Wehrlin et al. (1976) and Santolík et al. (2009).

The primary source region of chorus emissions lies in the vicinity of the magnetic equator (Dunckel and Helliwell, 1969; LeDocq et al., 1998; Santolík et al., 2005b, a), where the waves have a maximum amplitude near $L \approx 7$ (Li et al., 2011a; Meredith et al., 2012; Agapitov et al., 2013). The transverse coherence scale of the chorus-wave generation region on the basis of simultaneous multi-spacecraft measurements of the same chorus elements obtained by Cluster during 2002-2003 was found to be $\sim 200 \mathrm{~km}$ at $L \approx 4.4$ (Santolík and Gurnett, 2003), $~ 600 \mathrm{~km}$ at $L \approx 5$ (Agapitov et al., $2011 \mathrm{a}$ ) and $\sim 3000 \mathrm{~km}$ for $L \approx 8-9$ (Agapitov et al., 2010). A secondary source may lie in pockets of minimum magnetic field at high-latitude $\lambda$ in the dayside magnetosphere (Tsurutani and Smith, 1977; Keika et al., 2012) during high geomagnetic activity. However, this additional source region is beyond the scope of this study.

Chorus waves are generally thought to be generated by cyclotron resonance with energetic electrons (roughly 1 to $100 \mathrm{keV}$ ) injected from the plasma sheet (Kennel and Petschek, 1966; Tsurutani and Smith, 1977). The discrete nature of large-amplitude chorus elements implies that their generation mechanism is nonlinear (Dysthe, 1971; Omura et al., 1991; Trakhtengerts, 1999). Although this mechanism is still the subject of intense research (see Nunn, 1974; Nunn et al., 1997; Katoh and Omura, 2007; Omura et al., 2008; Cully et al., 2011, and references therein), there is growing consensus that large-amplitude chorus elements originate in nonlinear interaction between electron gyration and wave propagation. Although previous models have successfully reproduced rising-tone elements using quasi-parallel choruswave propagation (Nunn et al., 1997; Katoh and Omura, 2007; Omura et al., 2008; Nunn et al., 2009), very few have been able to generate falling tones, and those only under the assumption of an unrealistic particle distribution (Lampe et al., 2010) or resulting in unobserved properties of fallers, such as an initial upward hook (Nunn et al., 2009). Recently two models have succeeded in producing falling tones in reasonable agreement with observations (Nunn and Omura, 2012; Soto-Chavez et al., 2014). Given that most falling-tone chorus are observed to be very oblique, Li et al. (2011b) suggested that the classical excitation mechanism used to explain rising tones may not be responsible for their generation.

Following this idea, Kurita et al. (2012) have performed a Poynting flux vector analysis of falling-tone chorus elements and have found that most falling tones propagate away from the magnetic equator. A recent statistical study (Li et al., 2013) has also shown that the vast majority of chorus Poynting flux vectors points away from the equator for $\lambda \leq 15^{\circ}$. However, this analysis has been carried out for relatively large-amplitude waves solely; thus the intensity of reflected falling-tone chorus waves is probably not sufficiently strong to be taken into account in these results, as stated in $\mathrm{Li}$ et al. (2013).

Understanding how and why falling-tone chorus elements occur is of great value for models of particle acceleration in radiation belts. Nonlinear resonant interaction of strong whistler waves with electrons can be indeed responsible for very rapid energy gain. This process is quite sensitive to frequency modulation of wave amplitude, i.e., to the sign of $\mathrm{d} \omega / \mathrm{d} t$ (see, e.g., Tao et al., 2012, and references therein).

Recent work (see, e.g., Bortnik et al., 2006, 2007; Chen et al., 2009; Watt et al., 2012; Breuillard et al., 2012a, b; Chen et al., 2013) has demonstrated the robustness of the ray-tracing technique for determining chorus-wave propagation properties outside the plasmasphere. In this region, suprathermal electron fluxes increase with increasing $K_{p}$ and $L$-shell, and are larger on the nightside compared to dayside (see, e.g., Li et al., 2010). Similar tendencies have been found for Landau damping of chorus waves using ray-tracing simulations (Chen et al., 2013). Thus, for relatively small $L$-shells (outside the plasmapause) in the dayside magnetosphere during quiet times, chorus-wave propagation in cold plasma is acceptable as a first approximation. Ray tracing in cold plasma has indeed enabled successful studies of magnetospherically reflected chorus waves generated at the equator (Bortnik et al., 2008, 2009, 2011a, b; Agapitov et al., 2011c), whose spacecraft observations are scarce (Parrot et al., 2003, 2004; Agapitov et al., 2011c).

In this paper, we investigate the origin of falling-tone elements by means of the ray-tracing technique, taking as a basis their statistical characteristics observed by $\mathrm{Li}$ et al. (2011b, 2012).

\section{Modeling chorus elements in the source region}

To model generation of falling-tone chorus elements in the inner magnetosphere, we use a 3-D ray-tracing code (for details see Breuillard et al., 2012a, b) that includes a realistic 
model of the Earth's inner magnetosphere (Global Core Plasma Model: GCPM 2.2; see Gallagher et al., 2000). The magnetic field and thermal plasma density parameters chosen (Magnetic Local Time (MLT) $=09: 00$ and $K_{p}=4$; see Agapitov et al., 2011b; Breuillard et al., 2012b) are typical of the period in which most chorus waves occur in the inner magnetosphere. The electron density model employed in this study with the parameters described in the previous sentence is presented in Fig. 1. The key parameter, the plasmapause, is here clearly defined at $L=4$. The chorus source region is here assumed to be at the magnetic equator $(Z=0)$ at $X \sim 7$. We thus use the database of ray trajectories generated at the magnetic equator described in Breuillard et al. (2012b), but with an extended range of $L$-shells $\left(5 \leq L_{0} \leq 10\right.$; the index 0 indicates the equatorial chorus source region), yielding a total of $\sim 70000$ ray trajectories.

Each discrete chorus element (hereafter referred to as a single chorus element, an SCE) is modeled by a set of rays injected at the equator with initial parameters based on typical rising-tone chorus element characteristics. Thus, the frequency range chosen is $0.25 \leq \omega \leq 0.45 \Omega_{e, 0}$ (see Li et al., $2011 \mathrm{~b}, 2012$ ) with a step $\Delta \omega=0.05 \Omega_{e, 0}$. The initial wavenormal angle range is $5^{\circ} \leq \theta_{0} \leq 40^{\circ}$ (Agapitov et al., 2012, 2013) with $\Delta \theta_{0}=5^{\circ}$. The initial azimuthal range is $0^{\circ} \leq$ $\varphi_{0} \leq 180^{\circ}$, where $\varphi_{0}=180^{\circ}$ indicates that $k$ is initially directed towards the Earth with $\Delta \varphi_{0}=10^{\circ}$. Taking as a basis the transverse coherence scale results described in the previous section, the observed size of individual rising-tone elements seems to increase with increasing $L$-shell, and the roughly estimated realistic size of individual rising tones in the considered source region here $(L \approx 7)$ would be of the order of $2000 \mathrm{~km}$. Therefore we use in this study a realistic source size of $0.3 R_{\mathrm{E}}(\sim 2000 \mathrm{~km})$ wide in $L$-shell to model each individual rising-tone element. The wave power dependence of each SCE on $L_{0}$ is not uniform however, and we approximate this distribution by a Gaussian function with variance $\sim 0.3 R_{\mathrm{E}}$.

The wave power distribution for each SCE in the chorus source region must also be reproduced accurately. The equatorial chorus azimuthal angle distribution is assumed to be uniform. Thus, weighting is divided into three individual components representing the dependence on $\omega, L_{0}, \theta_{0}$. Chorus-wave power distribution in frequency is usually approximated by a Gaussian function with a maximum value of $\omega \approx 0.35 \Omega_{e, 0}$ (Burtis and Helliwell, 1976). For the MLT and $K_{p}$ parameters considered, the chorus-wave-normal distribution is presented in Agapitov et al. (2012), from which we obtain a function $h_{0}\left(\theta_{0}\right)$ representing the distribution of $\theta_{0}$ angles at the equator. Li et al. (2011a) have shown that the equatorial intensity of chorus waves at MLT $=09: 00$ for a moderate geomagnetic activity has a maximum in the $8 \leq L_{0} \leq 9$ range and reaches nearly 0 at $L_{0} \approx 5$.

Additionally, as stated above, each SCE is also modeled by a Gaussian function (of $\sim 1 R_{\mathrm{E}}$ width) weighting its wave power dependence on $L_{0}$. Hence, to properly model an SCE

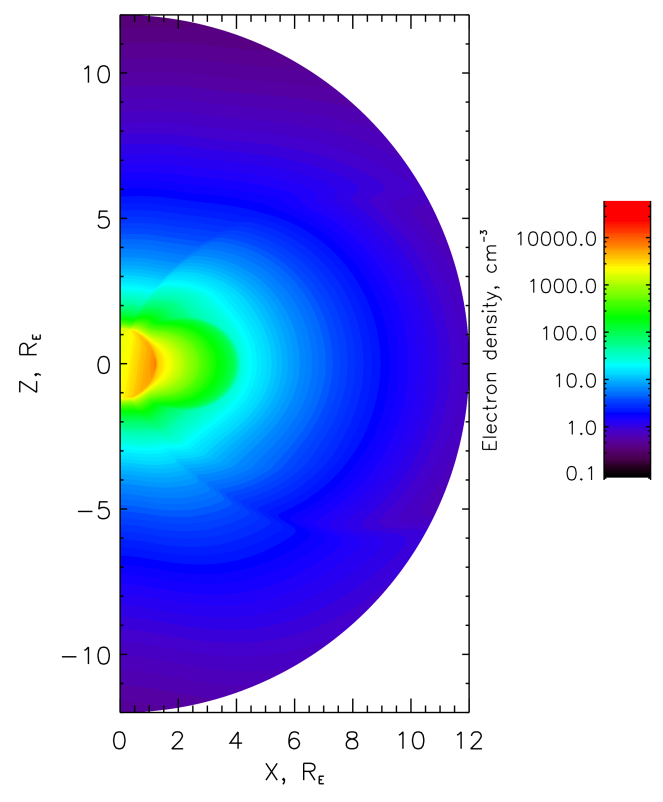

Figure 1. The employed model of electron density, given by the GCPM 2.2 model for the date 8 September 2002 at 05:00 UT, with MLT $=09: 00$ and $K_{p}=4$. The plasmapause is clearly defined here at $L=4$.

in the chorus source region, the global distribution of wave power must have a double dependence on $L_{0}$. As a result, each ray trajectory is weighted with an intensity given by the following weight function:

$$
\begin{aligned}
g\left(\theta_{0}, \omega_{0}, L_{0}\right)= & h_{0}\left(\theta_{0}\right) \exp \left[-\left(\frac{\left(L_{0}-L_{\mathrm{m}}\right)^{2}}{2(0.2)^{2}}\right)\right] \\
& \times \exp \left[-\left(\frac{\left(L_{0}-8.5\right)^{2}}{2(1.2)^{2}}\right)\right] \\
& \times \exp \left[-\left(\frac{\left(\omega_{0}-0.35 \Omega_{e, 0}\right)^{2}}{2\left(0.1 \Omega_{e, 0}\right)^{2}}\right)\right],
\end{aligned}
$$

where $L_{\mathrm{m}}$ is the $L_{0}$ value corresponding to the wave power distribution maximum for each SCE.

\section{Transformation of rising-tone elements into falling tones}

All SCEs are numerically injected at the equator and propagated through the inner magnetosphere. We obtain their properties at every point in space, notably when they recross the equator after magnetospheric reflection, after which ray tracing is stopped. Results of our modeling are shown in Fig. 2, in which one SCE is recorded in two different $L$-shells at the equator: in its injection region and in a lower $L$-shell. The upper panels in Fig. 2 show the time-frequency spectrograms obtained at $L=7$ and $L=5.5$ in the equatorial plane. In the upper-left panel, the dashed black line represents half the equatorial electron gyrofrequency $\Omega_{e, \text { equ }}$ in that particular 


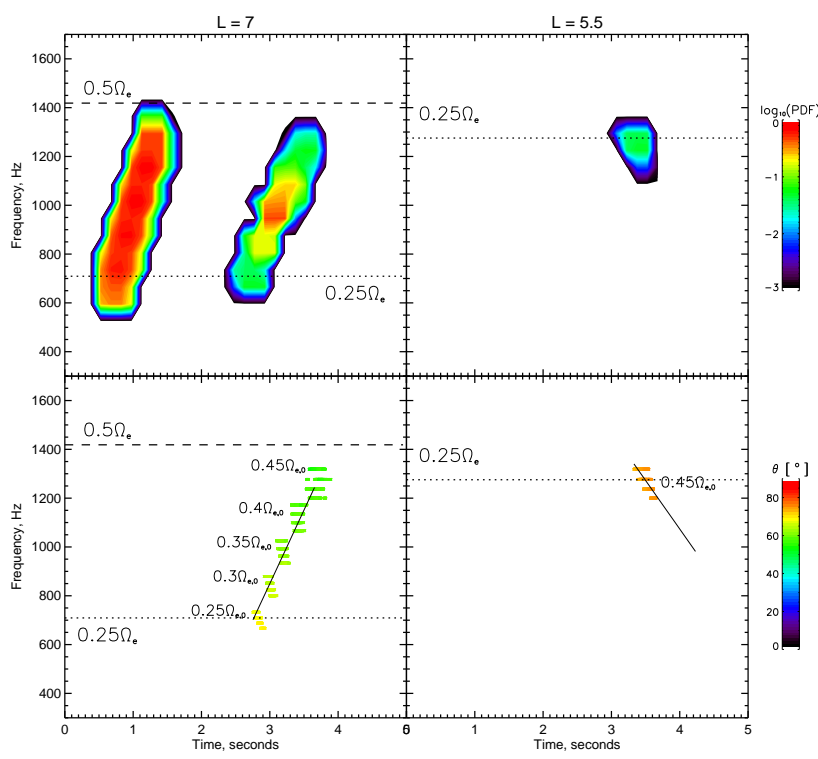

Figure 2. Time-frequency power spectra (top panels) of source and reflected chorus elements and their internal structure (bottom panels) as a function of $\theta$ angle (color), obtained from numerical simulations, recorded at the equator in two different regions $L=7$ (source) and $L=5.5$. In each panel, the dashed black line represents $0.5 \Omega_{e}$ and the dotted line represents $0.25 \Omega_{e}$. Initial ray frequencies normalized to $\Omega_{e, 0}$ are shown by text. The solid black line shows the sweep rate of each reflected element.

region, and the dotted line in both upper panels represents $0.25 \Omega_{e, \text { equ }}$.

In the upper-left panel, the injection region of the SCE, one can see the initial rising-tone element injected at $\sim 1 \mathrm{~s}$, as well as its reflected signal obtained in the same region at $\sim 3 \mathrm{~s}$. The reflected signal exhibits an approximately 520 times lower intensity than the initial signal due to spatial dispersion of ray trajectories during wave packet propagation and reflection (see Breuillard et al., 2013). However, both initial and reflected elements in this region exhibit similar characteristics, in particular, a rising tone $(\mathrm{d} \omega / \mathrm{d} t>0)$. The frequency sweep rate $\mathrm{d} \omega / \mathrm{d} t$, indicated by the solid black line in the bottom panels in this figure, is obtained by a linear fit of all points constituting each reflected element.

The reflected signal obtained in the lower $L$-shell region (upper-right panel), however, has characteristics that are completely different from those of the initial SCE. It exhibits a narrower bandwidth (about half the initial signal) centered around $0.25 \Omega_{e \text {,equ }}$ and a much ( $\sim 20-50$ times) lower intensity than the initial signal. Moreover, this signal mainly shows a falling tone $(\mathrm{d} \omega / \mathrm{d} t<0$, indicated by the solid black line in the bottom-right panel), which implies that falling-tone chorus elements can be obtained from magnetospherically reflected rising-tone chorus elements generated at higher $L$-shells.
The characteristics of the obtained reflected elements can be explained by spatiotemporal dispersion of the different ray trajectories, shown by the internal structure of the signals in the lower panels of Fig. 2. Technically, each point in the lower panels of Fig. 2 is a ray that has been reflected to $L=7$ and $L=5.5$, and the density of rays (weighted by their amplitude) is used to create the contours in the top panels, using small interpolation for the sake of clarity. The reflected signal in the SCE source region (lower-left panel) is composed of rays that deviate very little $\left( \pm 0.1 R_{\mathrm{E}}\right)$ from their initial magnetic field line. Thus, it includes all initial ray frequencies $\left(0.25-0.45 \Omega_{e, 0}\right.$, with $\Omega_{e, 0}$ being the electron gyrofrequency in the source region). Rays in this region are oblique $\left(40^{\circ} \leq \theta \leq 70^{\circ}\right)$, and higher $\theta$ predominates in the lower frequencies $\left(0.25-0.3 \Omega_{e, 0}\right)$ because rays with these frequencies reach higher $L$-shells during propagation. Hence, for these frequencies, only higher initial wave-normal angles $\theta_{0}$ are reflected at lower $L$-shells, yielding more oblique rays than higher $\Omega_{e, 0}$. Each set of reflected rays composing an initial frequency forms a characteristic "whistler-like" dispersive pattern, each horizontal line of which is a different $L_{0}$ because the gyrofrequency is different for each $L_{0}$ (higher lines thus indicate lower $L$-shells and vice versa). As a result, the presence of all initial frequencies gives a rising tone to the reflected element in the source region, and the sweep rate is even lowered by the time delay of rays with highest $\Omega_{e, 0}$ coming from lower $L$-shells.

To clarify the spatiotemporal dispersion of the different ray trajectories, in Fig. 3 we have selected four representative rays generated at different $L$-shells ( $L_{0}=6.8$ for upper row; $L_{0}=7.2$ for lower row) in the vicinity of the magnetic equator, and reflected to the equator in different regions $(L=7$ for left column; $L=5.5$ for right column). We first describe rays reflected at $L=7$. In the left column, rays are launched with a frequency $\omega=0.4 \Omega_{e, 0}$ from $L_{0}=6.8$ (panel a) and $L_{0}=7.2$ (panel c) with different parameters: $\theta=5^{\circ}$ and $\varphi=-180^{\circ}$ for panel a and $\theta=5^{\circ}$ and $\varphi=0^{\circ}$ for panel $\mathrm{c}$. It is clear that these two rays are reflected at $L \approx 7(X \approx$ $\left.4.4 \times 10^{7} \mathrm{~m}\right)$. However, their time of travel is different, and the first ray (launched at $L_{0}=6.8$ ) is delayed by about $0.22 \mathrm{~s}$ compared to the ray in panel c (launched at $L_{0}=7.2$ ), because after reflection it reaches higher $L$-shells. Ray a corresponds to a frequency of $\sim 1200 \mathrm{~Hz}$ in the bottom-left panel of Fig. 2, whereas ray c has a frequency $f \approx 1000 \mathrm{~Hz}$, because ray a is generated at $L=6.8$, where gyrofrequency is higher than at $L=7.2$. Thus, among rays with frequency $\omega=0.4 \Omega_{e, 0}$, high frequencies arrive after low frequencies, forming a rising tone. The sweep rate is even lowered by the presence of low frequencies $\left(0.25-0.35 \Omega_{e, 0}\right)$, which are generated before high frequencies $\left(0.4-0.45 \Omega_{e, 0}\right)$ in the wave packet, giving a clearly rising tone with a large bandwidth $(\sim 600 \mathrm{~Hz})$.

Then we describe the spatiotemporal dispersion of rays reflected at $L=5.5$. To do so, two representative rays with frequency $\omega=0.45 \Omega_{e, 0}$ and reflected at $L=5.5$ are presented 

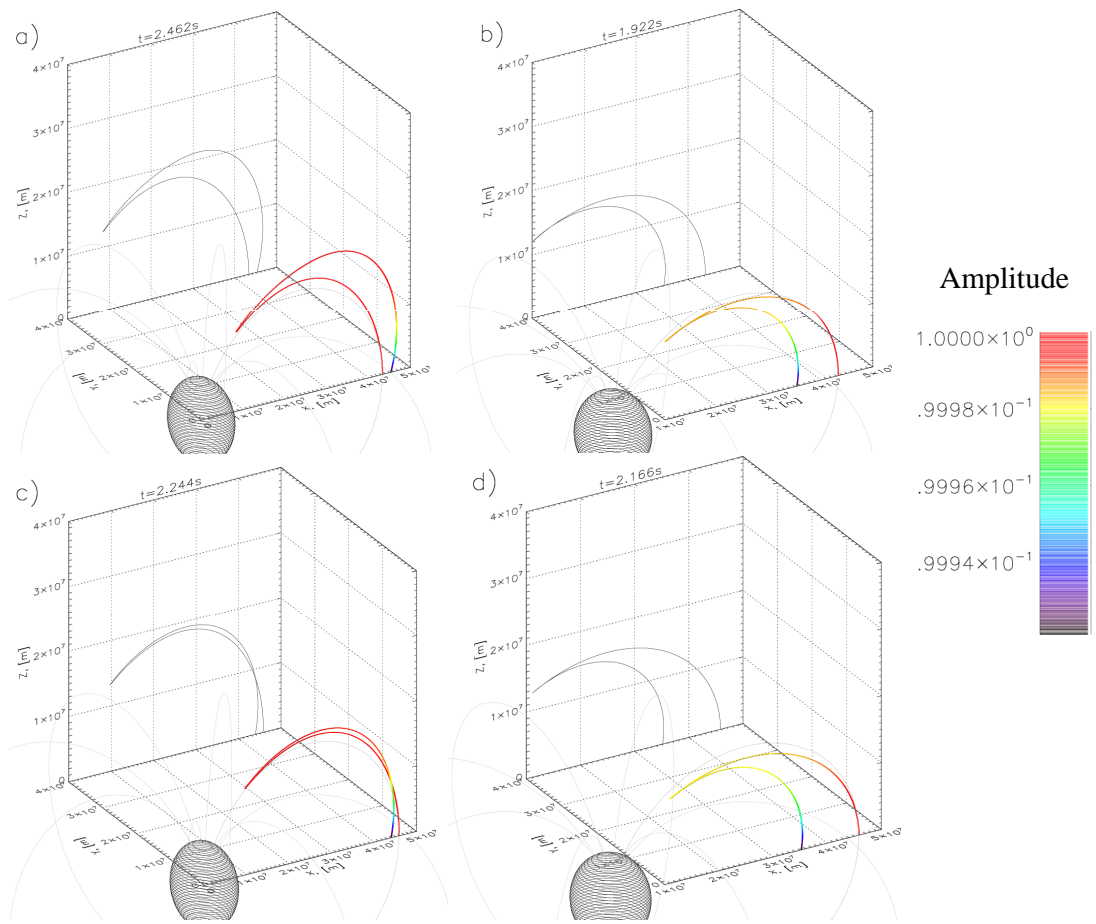

Figure 3. Sample trajectories of representative rays launched at $L=6.8$ (upper panels) and $L=7.2$ (lower panels), and reflected at $L=7$ (left panels) and $L=5.5$ (right panels). The ray's frequency is $\omega=0.4 \Omega_{e, 0}$ for rays a and c, and $\omega=0.45 \Omega_{e, 0}$ for rays b and d. The ray's time of travel $t$ is indicated in each panel. Color corresponds to the ray's amplitude (see colorbar), starting at the normalized value of 1 (red color) and being damped during their propagation back to the equator (blue color). The Earth is depicted in grey color.

in the right column. Ray $\mathrm{b}$ is launched at $L_{0}=6.8$ with $\theta=15^{\circ}$ and $\varphi=0^{\circ}$, and ray $\mathrm{d}$ is launched at $L_{0}=7.2$ with $\theta=30^{\circ}$ and $\varphi=0^{\circ}$. Here ray $\mathrm{d}$ reaches very low $L$-shells and its travel time is delayed by $\sim 0.24 \mathrm{~s}$ compared to ray $\mathrm{b}$, because its trajectory is longer. Ray b corresponds to a frequency of $\sim 1350 \mathrm{~Hz}$ in Fig. 2, whereas ray d corresponds to a frequency of $\sim 1150 \mathrm{~Hz}$, because gyrofrequency is lower at $L_{0}=7.2$ as stated above. As a result, in the bottom-right panel of Fig. 2, among rays with frequency $\omega=0.45 \Omega_{e, 0}$ low frequencies arrive after high frequencies; thus a falling tone is formed. Low frequencies $\left(0.25-0.35 \Omega_{e, 0}\right)$ generated in the wave packet are not reflected in this lower $L$ shell region; thus they are removed from the spectrum and the bandwidth and duration of the falling tone are reduced $(\sim 200 \mathrm{~Hz})$.

The reflected signal in the lower $L$-shell region (lowerright panel), however, is composed of only high frequencies $\left(0.35-0.45 \Omega_{e, 0}\right)$. Thus, it exhibits a lower intensity and a narrower bandwidth because the lower frequencies in the initial element are not reflected into such lower $L$-shells. At $L=5.5$, rays are also very oblique $\left(\theta \approx 75^{\circ}\right)$ because at these high $\Omega_{e, 0}$ only rays with higher $\theta_{0}$ have access to this region after their reflection. Therefore, in these regions the time delay of rays originating from higher $L$-shells and the absence of lower frequencies result in reversal of the sweep rate. Thus, we observe the formation of very oblique falling-tone elements with a narrow bandwidth. Since this phenomenon is only possible at $L$-shells significantly lower than $L_{0}$, the carrier frequency of the falling-tone element is lower than the local electron gyrofrequency $\left(\sim 0.25 \Omega_{e \text {,equ }}\right)$.

\section{Statistical properties of modeled falling-tone elements}

Making use of the technique described in the previous section for each rising-tone chorus element, we determine the statistical properties of the falling tones obtained by our raytracing simulations.

The database of ray trajectories used here covers the $5 \leq$ $L_{0} \leq 10$ range with a step $\Delta L_{0}=0.1 R_{\mathrm{E}}$, allowing us to propagate about 35 rising-tone elements $0.3 R_{\mathrm{E}}$ wide, each including $\sim 8000$ rays. Each ray is launched from the equator and propagated in the core $(0.5 \mathrm{eV})$ plasma at higher latitudes, where it can eventually reflect. We record each reflected ray trajectory when it recrosses the equator in the range $3 \leq L \leq 10$ with a bin size of $0.2 R_{\mathrm{E}}$ in $L$-shell and $1^{\circ}$ in latitude. In each bin, the points of the reflected element are fitted using a linear model to determine whether the tone is rising (positive frequency sweep rate) or falling (negative frequency sweep rate). The ray tracing is stopped when the rays recross the equator after their first reflection at high latitude. Thus, we trace the rays through the equator 
only once. Because ray trajectories disperse into different $L$ shells during propagation and reflection, we obtain about five falling tones (each is thus observed at different $L$ ) per rising tone launched at the equator. Using this technique, we obtain a total of about 180 falling-tone elements, the statistical distributions of which are compared with initial rising-tone properties and statistical distributions measured by THEMIS spacecraft (Li et al., 2011b, 2012).

Figure 4 shows the wave-normal angle, $L$-shell, frequency probability distribution functions obtained for modeled falling-tone chorus elements (in black), and modeled initial rising-tone chorus elements (in red). The various equatorial distributions for initial rising tones are described by the terms in Eq. (1); their choice is discussed in Sect. 2.

The wave-normal angle distribution (left panel) of fallingtone elements, a narrow Gaussian-like function centered at about $\theta_{\max } \approx 70^{\circ}$, is in good agreement with the Li et al. $(2011 b, 2012)$ results. This distribution indicates that, as observed previously (e.g., Cornilleau-Wehrlin et al., 1976; Santolík et al., 2009), falling tones propagate near the resonance cone even at the magnetic equator.

The modeled $L$-shell distribution of falling tones (middle panel) is also a Gaussian-like function centered around $L_{\max }=6.5$, which maximum is observed by Li et al. (2012) as well; thus, its maximum is shifted $\sim 2 R_{\mathrm{E}}$ inward of the rising-tone $L$ distribution maximum $\left(L_{\max }=8.5\right.$ for rising tones). This result is consistent with the observed confinement of falling tones to lower $L$-shells than rising tones $(\mathrm{Li}$ et al., 2011b). It is also in very good agreement with the statistical results obtained by $\mathrm{Li}$ et al. (2012) in the prenoon sector, where the maximum $L$ distribution of falling tones is shifted $\sim 2 R_{\mathrm{E}}$ inward of the rising-tone maximum.

The frequency distribution of modeled falling tones (right panel) is also consistent with most previous observations (see, e.g., Chum et al., 2009; Li et al., 2011b, 2012), since its bandwidth is half as wide $\left(\sim 0.1 \Omega_{e}\right.$,equ $)$ as that of rising tones $\left(\sim 0.2 \Omega_{e, \text { equ }}\right)$, and its central frequency is lower $\left(\sim 0.25 \Omega_{e, \text { equ }}\right)$ than the corresponding value for rising tones $\left(\sim 0.35 \Omega_{e, \text { equ }}\right)$.

\section{Discussion and conclusions}

In the present study, we modeled falling-tone chorus element properties using a 3-D ray-tracing code that includes a realistic model of Earth's inner magnetosphere. Rising-tone chorus elements with parameters typically observed onboard different spacecraft were injected at the equator in the chorus source region and propagated throughout the entire inner magnetosphere. We show that weak-amplitude fallingtone chorus elements can originate from magnetospheric reflection of rising tones generated at higher $L$-shells, due to the spatiotemporal dispersion of ray trajectories (see, e.g., Breuillard et al., 2013). The removal of lower frequencies from the spectra at lower $L$-shells and the time delay of
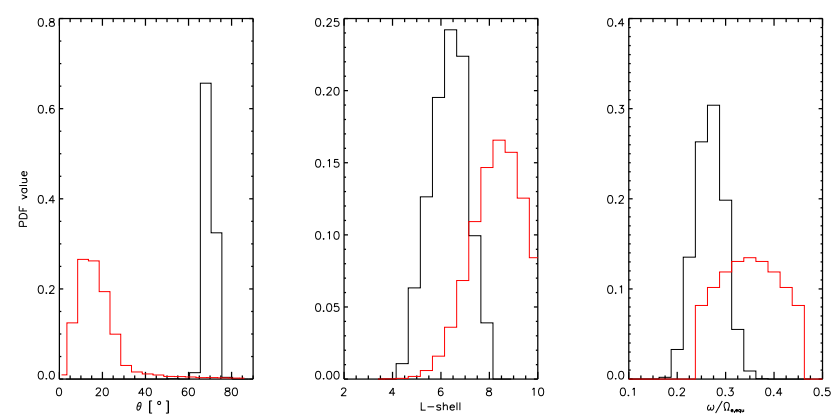

Figure 4. Probability distribution functions of wave-normal angle (left panel), $L$-shell (middle panel), and normalized frequency (right panel) obtained from numerical simulations of chorus elements. Statistical distributions of initial rising tones (red) and falling tones (black) are compared.

waves coming from higher $L$-shells indeed produce frequency sweep rate reversal. Thus, weak-amplitude falling tones can be viewed in our model as reflected and dispersed echoes of rising-tone emissions. The properties of fallingtone chorus elements found in this study are consistent with magnetic field measurements from THEMIS spacecraft presented in recent statistical distributions (e.g., Li et al., 2011b, 2012).

Li et al. (2011b) have suggested that the effects of wave propagation may play an important role in the evolution of chorus-wave elements. In our model, falling tones with a low frequency sweep rate are observed at much lower $L$ shells because at these $L$-shells more lower frequencies are removed from the spectrum and the time delay between rays from different $L$-shells is higher. Therefore, falling tones with low frequency sweep rate are observed farther from the original rising tone and thus exhibit a lower intensity, in agreement with THEMIS observations ( $\mathrm{Li}$ et al., 2011b, 2012). According to our model, the size of the source (rising tone) element affects the bandwidth and duration of the reflected falling tone; e.g., a smaller source size would give a smaller bandwidth and duration of the generated falling tones. Therefore, the wide range of bandwidths $(\sim 50-500 \mathrm{~Hz})$ and durations $(\sim 0.05-0.5 \mathrm{~s})$ of falling tones observed onboard spacecraft would be explained in terms of rising tone size variations $(100-3000 \mathrm{~km})$ in the source region. In addition, Li et al. (2011b) have shown that weakamplitude falling tones are observed mostly at $\lambda>10^{\circ}$ (see also Burtis and Helliwell, 1976). Our model is consistent with this result because falling tones are shown not to be generated at the equator as rising tones but to form during reflection at higher latitudes. Hence they are observed to propagate towards the magnetic equator. In fact, our model is able to form any type (rising and falling tones, hooks and structureless elements) of reflected chorus spectral structures observed onboard spacecraft (see, e.g., Agapitov et al., 2010), but this aspect is beyond the scope of this study and will be left for future investigation. 
According to Li et al. (2013) (see also references therein), reflected waves are observed to be at least 2 orders of magnitude weaker than source (equatorial) chorus. However, in this study the power spectra of reflected falling tones are about 1 order of magnitude weaker, because rays are propagated in background/core $(\sim 0.5 \mathrm{eV})$ plasma solely; thus the damping of waves is negligible $\left(\sim 10^{-4}\right)$ during their propagation. Therefore, according to our model the wave power decrease due to wave dispersion is about 1 to 1.5 orders of magnitude (see also Breuillard et al., 2013). However, the presence of energetic electrons $(\sim 0.1-100 \mathrm{keV})$ plays a crucial role in the wave damping/amplification during its propagation (see, e.g., Kennel and Thorne, 1967). Thus, if energetic electrons are taken into account in the model, waves would presumably grow at low latitudes $\left(\lambda<15^{\circ}\right)$ and then be damped at higher latitudes (see, e.g., Kennel and Thorne, 1967; Watt et al., 2012, 2013), and it is very likely that falling tones spectrum would be (taking into account wave dispersion and damping) at least 2 orders of magnitude weaker than source chorus, in accordance with Parrot et al. (2004), Santolík et al. (2010), and Li et al. (2013). In this sense, studies including a model of an outer radiation belt are currently conducted by the authors to estimate chorus-wave growth/damping during their propagation, in a realistic model of Earth's inner magnetosphere. Preliminary results seem to be consistent with this hypothesis.

As stated in the previous section, the frequency distribution of modeled falling tones (see right panel of Fig. 4) is also consistent with most previous observations (see, e.g., Chum et al., 2009; Li et al., 2011b, 2012) and all events analyzed in the frame of this study (not shown), since its peak frequency is lower than the corresponding value for rising tones. However, several falling-tone choruses have been observed (see Santolík et al., 2009; Kurita et al., 2012) to have a rather high peak frequency $\left(\sim 0.4 \Omega_{e \text {,equ }}\right)$ though their bandwidth is still rather narrow compared to typically observed rising tones. Therefore, a statistical study of observed falling-tone frequency distribution is needed to determine the global occurrence of their different bandwidths and peak frequencies. In addition, recently Nunn and Omura (2012) have shown, from a nonlinear generation model, that falling-tone chorus elements are not necessarily generated in the equatorial plane but can grow at some distance $(\sim 5200 \mathrm{~km})$ from the equator, and some can propagate towards the equator. Our model also suggests that weak-amplitude falling tones can be equatorward. Hence, measurements of falling-tone chorus elements should also be conducted to perform the identification and proportion of the different falling-tone populations and generation processes in the inner magnetosphere. A classification of these populations is needed to clarify their impact on energetic electron dynamics - particularly for $\lambda \geq 20^{\circ}$, where no falling tone measurements have been presented and should be subject to further investigation.

This paper thus provides a new model for the generation of weak-amplitude falling-tone chorus elements in the inner magnetosphere and emphasizes the significance of reflected chorus waves for the understanding of the inner magnetosphere dynamics (see also Santolík et al., 2010). Our model predicts that rising-tone chorus elements, which are generated at the equator and magnetospherically reflected to smaller $L$-shells, can turn into falling-tone elements that are then observed at lower latitudes. Therefore, these falling tones are confined to small $L$-shells and are found to be very oblique with a reduced frequency, bandwidth, and wave power, in good agreement with most previous observations (see, e.g., Cornilleau-Wehrlin et al., 1976; Li et al., 2011b, 2012, and references therein). This new model can thus be considered as a possible mechanism responsible for the generation of weak-amplitude falling-tone chorus, whereas relatively strong falling tones $(\geq \sim 5 \mathrm{pT})$, which are observed to propagate from the equator (Kurita et al., 2012; Li et al., 2013), may be generated by a different mechanism (e.g., Nunn and Omura, 2012; Soto-Chavez et al., 2014).

Acknowledgements. This work was supported by CNES through the grant "Modèles d'ondes".

Topical Editor C. Owen thanks C. Watt and one anonymous referee for their help in evaluating this paper.

\section{References}

Agapitov, O., Krasnoselskikh, V., Zaliznyak, Yu., Angelopoulos, V., Le Contel, O., and Rolland, G.: Chorus source region localization in the Earth's outer magnetosphere using THEMIS measurements, Ann. Geophys., 28, 1377-1386, doi:10.5194/angeo28-1377-2010, 2010.

Agapitov, O., Krasnoselskikh, V., Dudok de Wit, T., Khotyaintsev, Y., Pickett, J. S., Santolík, O., and Rolland, G.: Multispacecraft observations of chorus emissions as a tool for the plasma density fluctuations' remote sensing, J. Geophys. Res.-Space, 116, A09222, doi:10.1029/2011JA016540, 2011a.

Agapitov, O., Krasnoselskikh, V., Khotyaintsev, Y. V., and Rolland, G.: A statistical study of the propagation characteristics of whistler waves observed by Cluster, Geophys. Res. Lett., 38, L20103, doi:10.1029/2011GL049597, 2011b.

Agapitov, O., Krasnoselskikh, V., Zaliznyak, Yu., Angelopoulos, V., Le Contel, O., and Rolland, G.: Observations and modeling of forward and reflected chorus waves captured by THEMIS, Ann. Geophys., 29, 541-550, doi:10.5194/angeo-29-541-2011, 2011c.

Agapitov, O., Krasnoselskikh, V., Khotyaintsev, Y. V., and Rolland, G.: Correction to "A statistical study of the propagation characteristics of whistler waves observed by Cluster", Geophys. Res. Lett., 39, L24102, doi:10.1029/2012GL054320, 2012.

Agapitov, O., Artemyev, A., Krasnoselskikh, V., Khotyaintsev, Y. V., Mourenas, D., Breuillard, H., Balikhin, M., and Rolland, G.: Statistics of whistler-mode waves in the outer radiation belt: Cluster STAFF-SA measurements, J. Geophys. Res.-Space, 118, 3407-3420, doi:10.1002/jgra.50312, 2013.

Allcock, G. M.: A Study of the Audio-frequency Radio Phenomenon known as "Dawn Chorus", Aust. J. Physics, 10, 286297, doi:10.1071/PH570286, 1957. 
Anderson, R. R. and Maeda, K.: VLF emissions associated with enhanced magnetospheric electrons, J. Geophys. Res., 82, 135146, doi:10.1029/JA082i001p00135, 1977.

Bortnik, J., Inan, U. S., and Bell, T. F.: Landau damping and resultant unidirectional propagation of chorus waves, Geophys. Res. Lett., 33, L03102, doi:10.1029/2005GL024553, 2006.

Bortnik, J., Thorne, R. M., and Meredith, N. P.: Modeling the propagation characteristics of chorus using CRRES suprathermal electron fluxes, J. Geophys. Res.-Space, 112, A08204, doi:10.1029/2006JA012237, 2007.

Bortnik, J., Thorne, R. M., and Meredith, N. P.: The unexpected origin of plasmaspheric hiss from discrete chorus emissions, Nature, 452, 62-66, doi:10.1038/nature06741, 2008.

Bortnik, J., Li, W., Thorne, R. M., Angelopoulos, V., Cully, C., Bonnell, J., Le Contel, O., and Roux, A.: An Observation Linking the Origin of Plasmaspheric Hiss to Discrete Chorus Emissions, Science, 324, 775-778, doi:10.1126/science.1171273, 2009.

Bortnik, J., Chen, L., Li, W., Thorne, R. M., and Horne, R. B.: Modeling the evolution of chorus waves into plasmaspheric hiss, J. Geophys. Res.-Space, 116, A08221, doi:10.1029/2011JA016499, 2011a.

Bortnik, J., Chen, L., Li, W., Thorne, R. M., Meredith, N. P., and Horne, R. B.: Modeling the wave power distribution and characteristics of plasmaspheric hiss, J. Geophys. Res.-Space, 116, A12209, doi:10.1029/2011JA016862, 2011b.

Breuillard, H., Mendzhul, D., and Agapitov, O.: Effects of equatorial chorus wave normal azimuthal distribution on wave propagation, Adv. Astron. Space Phys., 2, 167-172, 2012a.

Breuillard, H., Zaliznyak, Y., Krasnoselskikh, V., Agapitov, O., Artemyev, A., and Rolland, G.: Chorus wave-normal statistics in the Earth's radiation belts from ray tracing technique, Ann. Geophys., 30, 1223-1233, doi:10.5194/angeo-30-1223-2012, $2012 \mathrm{~b}$.

Breuillard, H., Zaliznyak, Y., Agapitov, O., Artemyev, A., Krasnoselskikh, V., and Rolland, G.: Spatial spreading of magnetospherically reflected chorus elements in the inner magnetosphere, Ann. Geophys., 31, 1429-1435, doi:10.5194/angeo-311429-2013, 2013.

Burtis, W. J. and Helliwell, R. A.: Banded chorus - A new type of VLF radiation observed in the magnetosphere by OGO 1 and OGO 3, J. Geophys. Res., 74, 3002-3010, doi:10.1029/JA074i011p03002, 1969.

Burtis, W. J. and Helliwell, R. A.: Magnetospheric chorus - Occurrence patterns and normalized frequency, Planet Space Sci., 24, 1007-1024, doi:10.1016/0032-0633(76)90119-7, 1976.

Burton, R. K. and Holzer, R. E.: The Origin and Propagation of Chorus in the Outer Magnetosphere, J. Geophys. Res., 79, 10141023, doi:10.1029/JA079i007p01014, 1974.

Chen, L., Bortnik, J., Thorne, R. M., Horne, R. B., and Jordanova, V. K.: Three-dimensional ray tracing of VLF waves in a magnetospheric environment containing a plasmaspheric plume, Geophys. Res. Lett., 36, L22101, doi:10.1029/2009GL040451, 2009.

Chen, L., Thorne, R. M., Li, W., and Bortnik, J.: Modeling the wave normal distribution of chorus waves, J. Geophys. Res.-Space, 118, 1074-1088, doi:10.1029/2012JA018343, 2013.

Chum, J., Santolik, O., Gurnett, D. A., and Pickett, J. S.: Oblique lower band chorus waves: Time shifts between discrete elements observed by the Cluster spacecraft, J. Geophys. Res.-Space, 114, A00F02, doi:10.1029/2009JA014366, 2009.
Cornilleau-Wehrlin, N., Etcheto, J., and Burton, R. K.: Detailed analysis of magnetospheric ELF chorus - preliminary results, J. Atmos. Terr. Phy., 38, 1201-1210, 1976.

Cully, C. M., Angelopoulos, V., Auster, U., Bonnell, J., and Le Contel, O.: Observational evidence of the generation mechanism for rising-tone chorus, Geophys. Res. Lett., 38, L01106, doi:10.1029/2010GL045793, 2011.

Dunckel, N. and Helliwell, R. A.: Whistler-Mode Emissions on the OGO 1 Satellite, J. Geophys. Res., 74, 6371-6385, doi:10.1029/JA074i026p06371, 1969.

Dysthe, K. B.: Some studies of triggered whistler emissions, J. Geophys. Res., 76, 6915, doi:10.1029/JA076i028p06915, 1971.

Gallagher, D. L., Craven, P. D., and Comfort, R. H.: Global core plasma model, J. Geophys. Res., 105, 18819-18834, doi:10.1029/1999JA000241, 2000.

Hayakawa, M., Yamanaka, Y., Parrot, M., and Lefeuvre, F.: The wave normals of magnetospheric chorus emissions observed on board GEOS 2, J. Geophys. Res., 89, 2811-2821, doi:10.1029/JA089iA05p02811, 1984.

Katoh, Y. and Omura, Y.: Computer simulation of chorus wave generation in the Earth's inner magnetosphere, Geophys. Res. Lett., 34, L03102, doi:10.1029/2006GL028594, 2007.

Keika, K., Spasojevic, M., Li, W., Bortnik, J., Miyoshi, Y., and Angelopoulos, V.: PENGUIn/AGO and THEMIS conjugate observations of whistler mode chorus waves in the dayside uniform zone under steady solar wind and quiet geomagnetic conditions, J. Geophys. Res.-Space, 117, A07212, doi:10.1029/2012JA017708, 2012.

Kennel, C. F. and Petschek, H. E.: Limit on Stably Trapped Particle Fluxes, J. Geophys. Res., 71, 1, 1966.

Kennel, C. F. and Thorne, R. M.: Unstable growth of unducted whistlers propagating at an angle to the geomagnetic field, J. Geophys. Res., 72, 871-878, doi:10.1029/JZ072i003p00871, 1967.

Koons, H. C. and Roeder, J. L.: A survey of equatorial magnetospheric wave activity between 5 and 8 R(E), Planet Space Sci., 38, 1335-1341, doi:10.1016/0032-0633(90)90136-E, 1990.

Kurita, S., Misawa, H., Cully, C. M., Le Contel, O., and Angelopoulos, V.: Source location of falling chorus, Geophys. Res. Lett., 39, L22102, doi:10.1029/2012GL053929, 2012.

Lampe, M., Joyce, G., Manheimer, W. M., and Ganguli, G.: Nonlinear whistler instability driven by a beamlike distribution of resonant electrons, Phys. Plasmas, 17, 022902, doi:10.1063/1.3298733, 2010.

LeDocq, M. J., Gurnett, D. A., and Hospodarsky, G. B.: Chorus Source Locations from VLF Poynting Flux Measurements with the Polar Spacecraft, Geophys. Res. Lett., 25, 4063, doi:10.1029/1998GL900071, 1998.

Li, W., Thorne, R. M., Bortnik, J., Nishimura, Y., Angelopoulos, V., Chen, L., McFadden, J. P., and Bonnell, J. W.: Global distributions of suprathermal electrons observed on THEMIS and potential mechanisms for access into the plasmasphere, J. Geophys. Res.-Space, 115, A00J10, doi:10.1029/2010JA015687, 2010.

Li, W., Bortnik, J., Thorne, R. M., and Angelopoulos, V.: Global distribution of wave amplitudes and wave normal angles of chorus waves using THEMIS wave observations, J. Geophys. Res., 116, A12205, doi:10.1029/2011JA017035, 2011a.

Li, W., Thorne, R. M., Bortnik, J., Shprits, Y. Y., Nishimura, Y., Angelopoulos, V., Chaston, C., Le Contel, O., and Bonnell, 
J. W.: Typical properties of rising and falling tone chorus waves, Geophys. Res. Lett., 38, L14103, doi:10.1029/2011GL047925, 2011b.

Li, W., Thorne, R. M., Bortnik, J., Tao, X., and Angelopoulos, V.: Characteristics of hiss-like and discrete whistler-mode emissions, Geophys. Res. Lett., 39, L18106, doi:10.1029/2012GL053206, 2012.

Li, W., Bortnik, J., Thorne, R. M., Cully, C. M., Chen, L., Angelopoulos, V., Nishimura, Y., Tao, J. B., Bonnell, J. W., and LeContel, O.: Characteristics of the Poynting flux and wave normal vectors of whistler-mode waves observed on THEMIS, J. Geophys. Res.-Space, 118, 1461-1471, doi:10.1002/jgra.50176, 2013.

Meredith, N. P., Horne, R. B., Sicard-Piet, A., Boscher, D., Yearby, K. H., Li, W., and Thorne, R. M.: Global model of lower band and upper band chorus from multiple satellite observations, J. Geophys. Res.-Space, 117, A10225, doi:10.1029/2012JA017978, 2012.

Nunn, D.: A self-consistent theory of triggered VLF emissions, Planet Space Sci., 22, 349-378, doi:10.1016/00320633(74)90070-1, 1974.

Nunn, D. and Omura, Y.: A computational and theoretical analysis of falling frequency VLF emissions, J. Geophys. Res.-Space, 117, A08228, doi:10.1029/2012JA017557, 2012.

Nunn, D., Omura, Y., Matsumoto, H., Nagano, I., and Yagitani, S.: The numerical simulation of VLF chorus and discrete emissions observed on the Geotail satellite using a Vlasov code, J. Geophys. Res., 102, 27083-27098, doi:10.1029/97JA02518, 1997.

Nunn, D., Santolik, O., Rycroft, M., and Trakhtengerts, V.: On the numerical modelling of VLF chorus dynamical spectra, Ann. Geophys., 27, 2341-2359, doi:10.5194/angeo-27-23412009, 2009.

Omura, Y., Matsumoto, H., Nunn, D., and Rycroft, M. J.: A review of observational, theoretical and numerical studies of VLF triggered emissions, J. Atmos. Terr. Phy., 53, 351-368, 1991.

Omura, Y., Katoh, Y., and Summers, D.: Theory and simulation of the generation of whistler-mode chorus, J. Geophys. Res.-Space, 113, A04223, doi:10.1029/2007JA012622, 2008.

Parrot, M., Santolík, O., Cornilleau-Wehrlin, N., Maksimovic, M., and Harvey, C.: Magnetospherically reflected chorus waves revealed by ray tracing with CLUSTER data, Ann. Geophys., 21, 1111-1120, doi:10.5194/angeo-21-1111-2003, 2003.

Parrot, M., Santolík, O., Gurnett, D. A., Pickett, J. S., and Cornilleau-Wehrlin, N.: Characteristics of magnetospherically reflected chorus waves observed by CLUSTER, Ann. Geophys., 22, 2597-2606, doi:10.5194/angeo-22-2597-2004, 2004.

Santolík, O. and Gurnett, D. A.: Transverse dimensions of chorus in the source region, Geophys. Res. Lett., 30, 1031, doi:10.1029/2002GL016178, 2003.
Santolík, O., Gurnett, D. A., Pickett, J. S., Parrot, M., and Cornilleau-Wehrlin, N.: Central position of the source region of storm-time chorus, Planet Space Sci., 53, 299-305, doi:10.1016/j.pss.2004.09.056, 2005a.

Santolík, O., Macúšová, E., Yearby, K. H., Cornilleau-Wehrlin, N., and Alleyne, H. StC. K.: Radial variation of whistler-mode chorus: first results from the STAFF/DWP instrument on board the Double Star TC-1 spacecraft, Ann. Geophys., 23, 2937-2942, doi:10.5194/angeo-23-2937-2005, 2005b.

Santolík, O., Gurnett, D. A., Pickett, J. S., Chum, J., and CornilleauWehrlin, N.: Oblique propagation of whistler mode waves in the chorus source region, J. Geophys. Res.-Space, 114, A00F03, doi:10.1029/2009JA014586, 2009.

Santolík, O., Pickett, J. S., Gurnett, D. A., Menietti, J. D., Tsurutani, B. T., and Verkhoglyadova, O.: Survey of Poynting flux of whistler mode chorus in the outer zone, J. Geophys. Res.-Space, 115, A00F13, doi:10.1029/2009JA014925, 2010.

Sazhin, S. S. and Hayakawa, M.: Magnetospheric chorus emissions - A review, Planet Space Sci., 40, 681-697, doi:10.1016/00320633(92)90009-D, 1992.

Soto-Chavez, A. R., Wang, G., Bhattacharjee, A., Fu, G. Y., and Smith, H. M.: A model for falling-tone chorus, Geophys. Res. Lett., 41, 1838-1845, doi:10.1002/2014GL059320, 2014.

Storey, L. R. O.: An Investigation of Whistling Atmospherics, Philos T. R. Soc. A, 246, 113-141, doi:10.1098/rsta.1953.0011, 1953.

Tao, X., Bortnik, J., Thorne, R. M., Albert, J. M., and Li, W.: Effects of amplitude modulation on nonlinear interactions between electrons and chorus waves, Geophys. Res. Lett., 39, L06102, doi:10.1029/2012GL051202, 2012.

Trakhtengerts, V. Y.: A generation mechanism for chorus emission, Ann. Geophys., 17, 95-100, doi:10.1007/s00585-999-0095-4, 1999.

Tsurutani, B. T. and Smith, E. J.: Postmidnight Chorus: A Substorm Phenomenon, J. Geophys. Res., 79, 118-127, doi:10.1029/JA079i001p00118, 1974.

Tsurutani, B. T. and Smith, E. J.: Two types of magnetospheric ELF chorus and their substorm dependences, J. Geophys. Res., 82, 5112-5128, doi:10.1029/JA082i032p05112, 1977.

Watt, C. E. J., Rankin, R., and Degeling, A. W.: Whistler mode wave growth and propagation in the prenoon magnetosphere, J. Geophys. Res.-Space, 117, A06205, doi:10.1029/2012JA017765, 2012.

Watt, C. E. J., Degeling, A. W., and Rankin, R.: Constructing the frequency and wave normal distribution of whistlermode wave power, J. Geophys. Res.-Space, 118, 1984-1991, doi:10.1002/jgra.50231, 2013. 